\title{
Unusual gastric mucosal infiltration by a medullary thyroid carcinoma: a case report

\author{
T. Karrasch ${ }^{1^{*}} \mathbb{D}$, W. Doppl ${ }^{2}$, F. C. Roller ${ }^{3}$, A. Schäffler ${ }^{1}$, R. Schäffer ${ }^{4}$ and S. Gattenlöhner ${ }^{4}$
}

\begin{abstract}
Background: Medullary thyroid carcinoma accounts for approximately 1 to $2 \%$ of all thyroid carcinoma cases. The most common route of dissemination is to locoregional lymph nodes. Distant metastases commonly affect bones, lungs, and liver. We present a case of a white woman with a 25-year history of medullary thyroid carcinoma on multiple medications including tyrosine kinase inhibitor therapy for the last 11 months, who exhibited unusual diffuse infiltration of advanced stage medullary thyroid carcinoma to her gastric mucosa.

Case presentation: A 53-year-old white woman presented with increasing fatigue, loss of appetite, and severe epigastric pain radiating to her back. She had a history of medullary thyroid carcinoma (pT2pN1b), diagnosed 25 years ago and treated by complete thyroidectomy and repeated bilateral cervical lymph node dissection. Medical therapy included octreotide $20 \mathrm{mg}$ every 4 weeks, which was switched to the tyrosine kinase inhibitor vandetanib $300 \mathrm{mg} /$ day 11 months ago when computed tomography scanning revealed progressive mediastinal lymph node and diffuse and symptomatic pulmonary metastases. Of note, she demonstrated macroscopically stable pulmonary and mediastinal lymph node metastases; however, her calcitonin serum levels dramatically increased. Computed tomography scanning revealed a single new intrahepatic lesion $(4 \mathrm{~mm})$ as well as multiple (>10) new supraclavicular lesions suggestive of medullary thyroid carcinoma progress. As proven by gastric biopsy and immunohistochemical evaluation, her epigastric pain was explained by a diffuse infiltration of her gastric mucosa by metastatic medullary thyroid carcinoma. Subsequently, she rapidly deteriorated and died.

Conclusions: The current case report shows for the first time an unusual metastatic infiltration of the gastric mucosa by medullary thyroid carcinoma. When treating these patients, it is important to include this differential diagnosis during follow-up.
\end{abstract}

Keywords: Epigastric pain, Medullary thyroid carcinoma, Distant metastasis, Gastric mucosa, Tyrosine kinase inhibitors, Case report

\section{Background}

Thyroid cancer overall represents a rare tumor entity, however, the incidence of thyroid cancer has recently been constantly increasing $[1,2]$. Within this heterogeneous group of tumors, differentiated thyroid cancer represents the majority (approximately $90 \%$ ) of all newly diagnosed thyroid cancer cases [3]. Medullary thyroid carcinoma (MTC) accounts for approximately 1 to $2 \%$ of all thyroid carcinoma cases, and at diagnosis 7 to $23 \%$ of all patients demonstrate metastatic disease [1, 4]. Overall, 10-year disease-specific survival rates average approximately $75 \%$

\footnotetext{
* Correspondence: thomas.karrasch@innere.med.uni-giessen.de

${ }^{1}$ Department of Internal Medicine III, Giessen University Hospital, Klinikstrasse

33, 35392 Gießen, Germany

Full list of author information is available at the end of the article
}

with a strong dependency on tumor stage (averaging $21 \%$ in stage IV tumors). Metastases are commonly found in the lymph nodes, while distant metastatic disease is found in lungs, bone, and liver, and, more rarely, in brain, skin, and breast $[5,6]$. Therapeutically, the recent introduction of tyrosine kinase inhibitor therapy (vandetanib, cabozantinib) has led to a significant improvement of the prognosis in patients with rapidly progressive and advanced stage MTC [7].

\section{Case presentation}

A 53-year-old white woman presented with increasing fatigue, loss of appetite, and severe epigastric pain radiating to her back. She had a history of MTC (pT2pN1b), diagnosed 25 years ago and treated by complete 
thyroidectomy and bilateral cervical lymph node dissection, followed by repeated surgical resection of lymph node metastases 19 and 12 years ago. No further specific treatment modalities (for example radiotherapy) had been employed. No genetic analysis had been performed for germline or somatic RET proto-oncogene mutations, nor for other somatic mutations (HRAS, KRAS, NRAS). On clinical examination, however, there was no indication of other manifestations of multiple endocrine neoplasia type 2 (MEN2), especially no clinical signs of pheochromocytoma. Since data on the RET protooncogene status have been missing in our patient, no risk category (moderate, high, highest) according to the revised American Thyroid Association Guidelines for the management of MTC [1] had been assigned. However, based on the American Joint Committee on Cancer (AJCC) TNM-Classification, the MTC had been a stage IV A tumor (pT2pN1b) at the time of the initial complete thyroidectomy in our patient. Post complete thyroidectomy, she had hypoparathyroidism, which was treated with calcium and calcitriol. She received levothyroxine replacement therapy. Repeated biochemical and clinical evaluations during the course of the disease did not demonstrate any signs of paraneoplastic adrenocorticotropic hormone $(\mathrm{ACTH})$ and/or corticotropin-releasing hormone $(\mathrm{CRH})$ secretion. Medical therapy of the MTC included octreotide $20 \mathrm{mg}$ every 4 weeks, which was switched to the tyrosine kinase inhibitor vandetanib 300 $\mathrm{mg} /$ day 11 month ago when computed tomography $(\mathrm{CT})$ scanning revealed progressive mediastinal lymph node and diffuse and symptomatic pulmonary metastases.

Standard laboratory tests in our patient revealed moderate leukocytosis $(18.3 \mathrm{G} / \mathrm{l})$ as well as slightly elevated serum CRP levels $(12.58 \mathrm{mg} / \mathrm{l})$; her serum lactate was $5.5 \mathrm{mmol} / \mathrm{l}$. Pancreatitis was ruled out because her serum amylase and lipase levels were within normal range. An upper endoscopy was performed and demonstrated multiple centrally ulcerated lesions in her gastric mucosa of up to $4 \mathrm{~mm}$ in size (Fig. 1a, b). Two-phase contrast-enhanced multi-detector CT of her chest and abdomen revealed stable pulmonary and mediastinal lymph node metastases; however, there was a single new intrahepatic lesion of $4 \mathrm{~mm}$ size suspicious for liver metastasis. Contrast-enhanced multi-detector CT of her neck revealed multiple, new, centrally hypodense supraclavicular lesions up to $12 \mathrm{~mm}$ in size suggestive of lymph node metastases. In addition, multiple noduli within her stomach wall were apparent (Fig. 1c, I-III). Three-phase bone scintigraphy showed no evidence of bone metastasis.

Additional laboratory tests revealed a dramatic increase in her basal serum calcitonin levels from $563 \mathrm{pg} /$ $\mathrm{ml}$ to $6487 \mathrm{pg} / \mathrm{ml}$ within 2 months, indicative of rapid tumor progress of the underlying MTC. Of interest, at the same time she had rather stable carcinoembryonic antigen (CEA) serum levels of $40.7 \mathrm{ng} / \mathrm{ml}$ (from 62.3 $\mathrm{ng} / \mathrm{ml} 14$ months earlier). Histologic and immunohistochemical analyses of gastric biopsy specimen demonstrated diffuse mucosal infiltration of MTC (Fig. 1d-h) with a KI-67 index of up to $40 \%$, proving metastatic disease of MTC to the gastric mucosa. Of note, while immunohistochemical analysis of calcitonin was strongly positive (Fig. 1g), staining for CEA demonstrated merely focal and weak staining (Fig. 1h); staining for thyroglobulin remained completely negative (data provided in Additional file 1). She rapidly deteriorated and died due to multiorgan failure before third-line treatment could be initiated.

\section{Conclusions}

MTC is sporadic in origin in 60 to $75 \%$ of all patients with MTC, while the others exhibit germline mutations in the RET proto-oncogene, namely patients with multiple endocrine neoplasia type $2 \mathrm{~A}$ (MEN2A), multiple endocrine neoplasia type 2B (MEN2B), and familial MTC syndrome (FMTC). The most common sites for metastatic disease in MTC are locoregional lymph nodes; these metastases often occur early in the disease process [8]. This was also the case in our patient, who initially presented with a pT2pN1b tumor stage, and who received repeated resection of cervical lymph node metastases during the following years.

However, in advanced MTC, distant metastases have been described. In one report, $74 \%$ of patients demonstrated metastatic disease to the bone [9], other groups found small hepatic metastases in $25 \%$ of patients with advanced MTC [10], as well as lung metastases in $35 \%$ of patients with MTC with persistently elevated calcitonin levels after initial treatment [5]. In addition, cases of cutaneous [11, 12], brain [13], and breast [14, 15] metastases of MTC have been described.

Metastatic disease to the stomach overall is a rare incidence in all cancer types, and data in the literature are based on case reports as well as small case series [16-18]. The most common primary tumors reported to spread to the stomach are melanoma, breast, lung, and esophageal carcinoma as well as renal cell cancer [17]. On clinical examination, gastric metastases present with epigastric pain, nausea, vomiting, as well as gastrointestinal hemorrhage. Mostly solitary lesions within the gastric mucosa are observed, although multiple metastatic lesions have been reported as well [17]. To the best of our knowledge, metastatic disease to the gastric mucosa of a MTC has not yet been reported in the literature.

Our patient had MTC with a follow-up history of 25 years and presented with severe epigastric pain. Multiple 


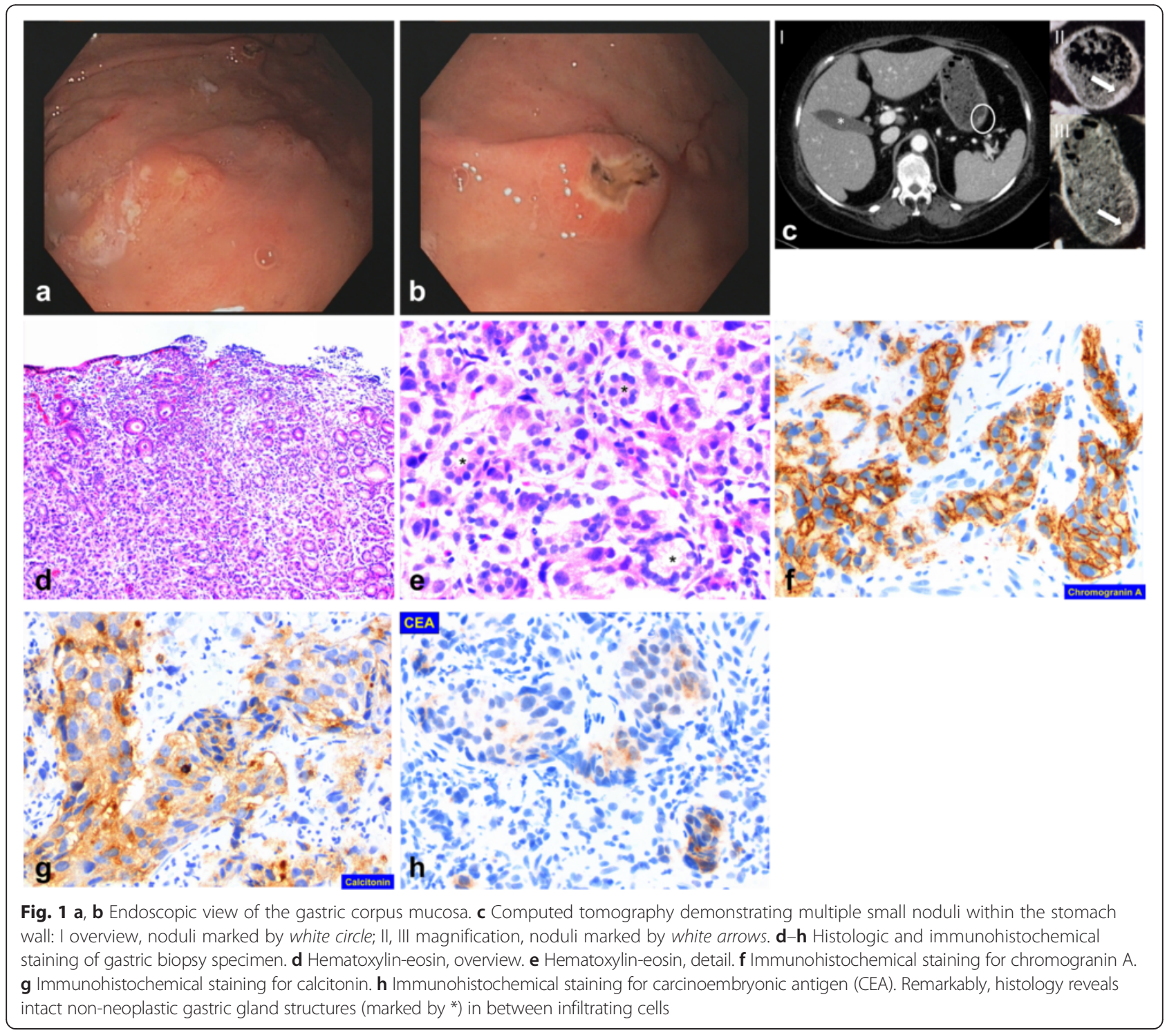

lesions within her gastric mucosa were histologically proved to be a diffuse metastatic infiltration of the MTC. Of note, CT scans of her chest and abdomen revealed stable pulmonary and mediastinal lymph node metastases; however, multiple supraclavicular lesions as well as a single new intrahepatic lesion were suggestive of MTC progress. Remarkably, a sharp rise in calcitonin levels was indicative of rapid tumor progression as well, while her serum levels of CEA remained rather stable.

The current case report should raise awareness of unusual gastrointestinal metastatic disease of MTC in advanced disease states, especially with the increasing prognosis of these tumors due to molecular-targeted oncologic therapies (for example tyrosine kinase inhibitors). In treating these patients, it is important to include this generally uncommon differential diagnosis for unspecific patient complaints during follow-up.

\section{Additional file}

Additional file 1: Immunohistochemical staining of gastric biopsy specimen for thyroglobulin remained completely negative. (TIF $1120 \mathrm{~kb}$ )

\section{Abbreviations}

ACTH, adrenocorticotropic hormone; AJCC, American Joint Committee on Cancer; CEA, carcinoembryonic antigen; $\mathrm{CRH}$, corticotropin-releasing hormone; CT, computed tomography; FMTC, familial medullary thyroid carcinoma syndrome; MEN2, multiple endocrine neoplasia type 2; MEN2A, multiple endocrine neoplasia type 2A; MEN2B, multiple endocrine neoplasia type 2B; MTC, medullary thyroid carcinoma

\section{Acknowledgements}

We thank the Department of Radiology, Giessen University Hospital, for preparation of the computed tomography scans presented in our manuscript.

\section{Funding}

No funding agency was involved in our patient's treatment or our preparation of the manuscript. 


\section{Availability of data and materials}

The dataset supporting the conclusions of this case report is included within the case report (images and laboratory parameters).

\section{Authors' contributions}

Acquisition, analysis, and interpretation of the data: TK, WD, FCR, AS, RS, and SG. Manuscript preparation: TK and AS. Critical revision and significant intellectual contribution to the manuscript: TK, WD, FCR, AS, RS, and SG. All authors read and approved the final manuscript.

\section{Competing interests}

The authors declare that they have no competing interests.

\section{Consent for publication}

Written informed consent was obtained from the patient's next-of-kin for publication of this case report and any accompanying images. A copy of the written consent is available for review by the Editor-in-Chief of this journal.

\section{Ethics approval and consent to participate}

Not applicable.

\section{Author details}

1 Department of Internal Medicine III, Giessen University Hospital, Klinikstrasse 33, 35392 Gießen, Germany. ${ }^{2}$ Central Interdisciplinary Endoscopy Unit (ZIVE), Giessen University Hospital, 35392 Gießen, Germany. ${ }^{3}$ Department of Radiology, Giessen University Hospital, 35392 Gießen, Germany. ${ }^{4}$ Department of Pathology, Giessen University Hospital, 35392 Gießen, Germany.

Received: 6 April 2016 Accepted: 28 June 2016

Published online: 27 July 2016

\section{References}

1. Wells Jr SA, Asa SL, Dralle H, Elisei R, Evans DB, Gagel RF, et al. Revised American Thyroid Association guidelines for the management of medullary thyroid carcinoma. Thyroid. 2015;25(6):567-610.

2. Radespiel-Troger M, Batzler WU, Holleczek B, Luttmann S, Pritzkuleit R, Stabenow $R$, et al. Rising incidence of papillary thyroid carcinoma in Germany. Bundesgesundheitsblatt Gesundheitsforschung Gesundheitsschutz. 2014;57(1):84-92.

3. American Thyroid Association Guidelines Taskforce on Thyroid Nodules and Differentiated Thyroid Cancer, Cooper DS, Doherty GM, Haugen BR, Kloos RT, Lee SL, et al. Revised American Thyroid Association management guidelines for patients with thyroid nodules and differentiated thyroid cancer. Thyroid. 2009;19(11):1167-214.

4. $\quad$ Schlumberger M, Bastholt L, Dralle H, Jarzab B, Pacini F, Smit JW, et al. 2012 European Thyroid Association guidelines for metastatic medullary thyroid cancer. Eur Thyroid J. 2012;1(1):5-14.

5. Giraudet AL, Vanel D, Leboulleux S, Auperin A, Dromain C, Chami L, et al. Imaging medullary thyroid carcinoma with persistent elevated calcitonin levels. J Clin Endocrinol Metab. 2007;92(11):4185-90.

6. Pacini F, Castagna MG, Cipri C, Schlumberger M. Medullary thyroid carcinoma. Clin Oncol. 2010;22(6):475-85.

7. Cabanillas ME, Hu MI, Jimenez C. Medullary thyroid cancer in the era of tyrosine kinase inhibitors: to treat or not to treat - and with which drug those are the questions. J Clin Endocrinol Metab. 2014;99(12):4390-6.

8. Maxwell JE, Sherman SK, O'Dorisio TM, Howe JR. Medical management of metastatic medullary thyroid cancer. Cancer. 2014;120(21):3287-301.

9. Mirallie E, Vuillez JP, Bardet S, Frampas E, Dupas B, Ferrer L, et al. High frequency of bone/bone marrow involvement in advanced medullary thyroid cancer. J Clin Endocrinol Metab. 2005:90(2):779-88.

10. Tung WS, Vesely TM, Moley JF. Laparoscopic detection of hepatic metastases in patients with residual or recurrent medullary thyroid cancer. Surgery. 1995;118(6):1024-9. discussion 1029-1030.

11. Nashed C, Sakpal SV, Cherneykin S, Chamberlain RS. Medullary thyroid carcinoma metastatic to skin. J Cutan Pathol. 2010;37(12):1237-40.

12. Ordonez NG, Samaan NA. Medullary carcinoma of the thyroid metastatic to the skin: report of two cases. J Cutan Pathol. 1987:14(4):251-4.

13. Borcek P, Asa SL, Gentili F, Ezzat S, Kiehl TR. Brain metastasis from medullary thyroid carcinoma. BMJ case reports. 2010;2010: bcr0920103301. doi:10.1136/bcr.09.2010.3301
14. Marcy PY, Thariat J, Peyrottes I, Dassonville O. Bilateral breast involvement in medullary thyroid carcinoma. Thyroid. 2009;19(2):197-9.

15. Mandanas S, Margaritidou E, Christoforidou V, Karoglou E, Geranou C, Chrisoulidou A, et al. Breast metastasis from medullary thyroid carcinoma in a male patient: case report and review of the literature. Rare Tumors. 2015;7(2):5765.

16. De Palma GD, Masone S, Rega M, Simeoli I, Donisi M, Addeo P, et al. Metastatic tumors to the stomach: clinical and endoscopic features. World J Gastroenterol. 2006;12(45):7326-8.

17. Namikawa T, Hanazaki K. Clinicopathological features and treatment outcomes of metastatic tumors in the stomach. Surg Today. 2014;44(8):1392-9.

18. Weigt J, Malfertheiner P. Metastatic disease in the stomach. Gastrointestinal tumors. 2015:2(2):61-4

\section{Submit your next manuscript to BioMed Central and we will help you at every step:}

- We accept pre-submission inquiries

- Our selector tool helps you to find the most relevant journal

- We provide round the clock customer support

- Convenient online submission

- Thorough peer review

- Inclusion in PubMed and all major indexing services

- Maximum visibility for your research

Submit your manuscript at www.biomedcentral.com/submit
Biomed Central 